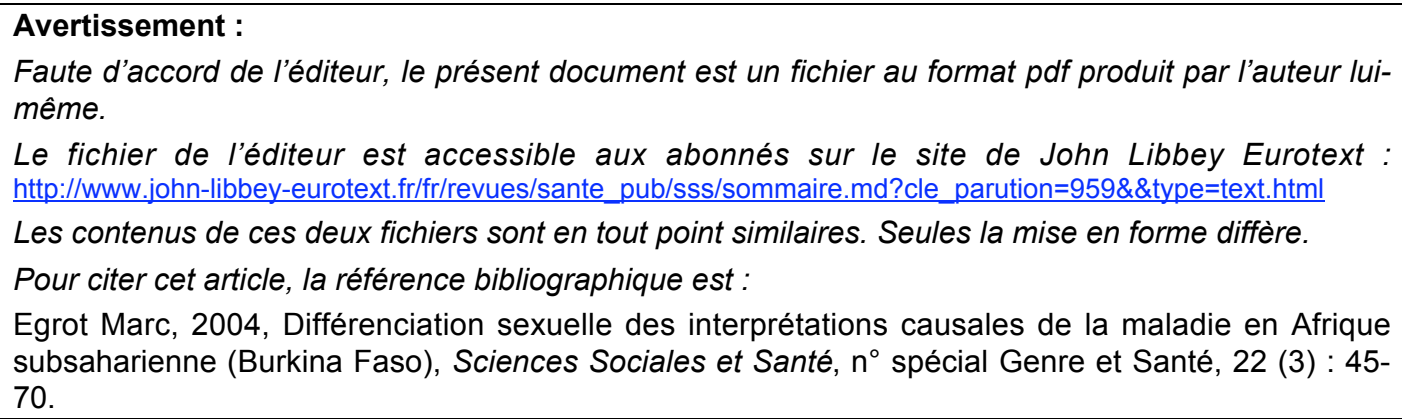

Sciences Sociales et Santé, Vol. 22, $n^{\circ} 3$, septembre $2004:$ 45-70.

\title{
Différenciation sexuelle des interprétations causales de la maladie en Afrique subsaharienne (Burkina Faso)
}

Marc Egrot*

Résumé. Le sexe social intervient dans la construction de la représentation de plusieurs maladies chez les Mossi du Burkina Faso, en particulier celles qui concernent la sexualité (maladies transmises lors d'un rapport sexuel), la reproduction individuelle (infécondité, impuissance) et la reproduction du groupe (maladies des enfants - propriété du patrilignage - et sorcellerie). Lorsqu'elles existent, ces interférences se traduisent principalement par une asymétrie dans les modèles étiologiques et les logiques d'imputation et sont autant d'occasions de faire valoir les normes sociales associées à chaque sexe et les risques engendrés par leur transgression. La maladie apparaît donc comme l'un des lieux d'expression mais également comme un espace de légitimation de la différenciation sociale des sexes.

Mots-clés : sexe social, maladie, étiologie, logiques d'imputation, Mossi, Burkina Faso.

\footnotetext{
* Marc Egrot, médecin, anthropologue, Centre de Recherche Cultures, Santé, Sociétés (CreCss), PAS, MNSH, 5, rue du Château de l'Horloge, 13094, Aix-en-Provence Cedex 2, France.
} 
La différence entre les sexes en matière de santé est un fait épidémiologique attesté et bien documenté ces dernières années ${ }^{1}$. Des différences de morbidité entre hommes et femmes existent pour de nombreuses maladies. Souvent, des raisons sociales ou culturelles en sont la cause (Annandale et Hunt, 2000 ; Pollard et Hyatt, 1999 ; Sargent et Brettell, 1995). Ainsi, des différences de tâche, de comportements, de régime alimentaire ou encore d'implication dans les soins domestiques expliquent parfois les écarts entre les deux sexes (Rao Gupta et Weiss, 1993 ; Saillant, 1991, 1992 ; Sweetman, 2001) Mais l'interférence entre sexe et maladie se fait aussi dans un autre registre qui oblige à explorer les représentations de la maladie, pour déboucher sur les usages sociaux (Zempleni, 1982) et l'espace politique de la santé (Fassin, 1996) ${ }^{2}$.

Peu après la reconnaissance officielle du sida en 1986 au Burkina Faso, les logiques d'imputations mobilisées pour cette maladie se sont orientées vers les femmes. Le sida est rapidement inséré dans une catégorie locale nommée " maladies de femmes ${ }^{3}$ avec un discours qui les désigne comme vecteurs de la transmission. De plus, la sémiologie populaire insiste sur le fait que la maladie débuterait plus tard, évoluerait plus lentement et serait moins grave chez les femmes (Egrot, 2001).

Certes, ces représentations ont été influencées par la prévention et les discours de divers acteurs de la lutte contre le sida, notamment sur la fidélité ou le lévirat (Taverne, 1996, 1999), ou encore par la stigmatisation de " groupes à risque », en particulier les prostituées (Desclaux, 1997).

\footnotetext{
${ }^{1}$ À titre d'exemple, citons la plus grande vulnérabilité des femmes au regard de la transmission du VIH lors des rapports sexuels (Mastro et de Vincenzi, 1996) ou encore la différence de prévalence des maladies cardiovasculaires ou de l'état nutritionnel (Pollard et Hyatt, 1999).

${ }^{2}$ Fassin aborde le sexe social à propos de «l'incorporation des inégalités » (Fassin, 1996 : 64-73). Cet article montrera que la différenciation des sexes émerge aussi dans la gestion collective de la santé (ce que Fassin intitule "le gouvernement de la vie »). La maladie apparaît en effet ici comme un moyen de renforcer ce qui, d'un point de vue émique, est interprété comme une nécessaire séparation des sexes. Du fait qu'elle permette de légitimer la différenciation sociale des sexes, la maladie est non seulement objet mais également instrument de pouvoir dans la société.

${ }^{3}$ Une dizaine de maladies qui ont en commun la possible transmission lors d'un rapport sexuel. Beaucoup d'infections sexuellement transmissibles (IST) de la biomédecine s'y retrouvent. Mais toute IST n'y est pas forcément incluse et une maladie de femmes n'est pas toujours une IST (voir plus loin et et Egrot et Taverne, 2003). Par ailleurs, cette catégorie existe dans bien d'autres ethnies en Afrique (voir, par exemple, Fainzang, 1986 ou Duchesne, 2003).
}

\section{Page 46}


Mais la rapidité avec laquelle cette logique d'imputation a émergé et l'existence d'une catégorie de " maladies de femmes » montrent que cette asymétrie des registres étiologiques n'a fait qu'entrer en résonance avec des conceptions déjà bien établies. Ce constat soulève alors une série d'interrogations.

Quelles sont les autres représentations de la maladie qui mobilisent une causalité orientée plutôt vers l'un ou l'autre sexe ? Y-a-til d'autres types d'interférences entre modèles explicatifs et sexe social ${ }^{4}$ ? Quels éléments culturels permettent de construire une telle asymétrie sexuelle des registres étiologiques ? Enfin, ces logiques de causalité donnent un sens particulier aux événements concernés et il est alors légitime de s'interroger sur les usages sociaux et culturels qui doivent nécessairement en découler. Cet article propose d'explorer les représentations de la maladie chez les Moose, puis d'apporter des réponses aux questions qui viennent d'être soulevées.

\section{Le sexe social : mode de déclinaison de la maladie}

\section{Troubles des fonctions reproductrices}

Chez les Moose, différentes interprétations expliquent la conception d'un enfant. L'une relève d'une représentation de la physiologie : l'« eau de sexe " de la femme, " versée " en premier, est aspirée par la " bouche de la verge » où elle rencontre "l'eau de l'homme ", créant un " mélange " qui sera " versé » à son tour (Egrot et Taverne, 2003). Un registre symbolique interprète " la procréation (comme) le résultat de la pénétration d'un génie (kinkirga) dans le ventre de la femme au moment où celle-ci a un rapport sexuel » (Bonnet, 1988). Mais les génies ne suffisent pas à la production d'êtres humains, en particulier pour l'une des composantes de la personne nommée siiga qui nécessite l'apport d' " une parcelle ontologique d'ancêtre ", le sèègre, issu lui-même du morcellement du siiga d'un aïeul (Bonnet, 1988). Ces interprétations, mobilisées de manière diachronique ou synchronique, apparaissent complémentaires pour deux raisons. Le génie entre dans le corps de la femme durant la pénétration de l'homme et la formation du foetus se fait grâce à " la masse de sang " issue des " eaux de sexe ", dont celle de l'homme qui apporte les caractères du patrilignage.

\footnotetext{
${ }^{4}$ Pour des raisons trop longues à reprendre ici (voir Egrot, 2001 : 45-52), je préfère utiliser le terme « sexe social » plutôt que « genre » ou gender.
} 
La fécondité reste néanmoins une qualité exclusivement féminine. En plus des travaux déjà publiés (Badini, 1978 ; Bonnet, 1998), il suffirait, pour s'en convaincre encore, d'explorer l'étymologie de termes variés pour lesquels le suffixe raogo (mâle) est ajouté pour des végétaux n'ayant pas de fruits ou pour cette phase du cycle lunaire, la "lune-mâle ", pendant laquelle rien ne doit être entrepris sous peine d'échec, notamment dans les domaines de la procréation ou de l'agriculture. Mais, bien qu'une infécondité ne puisse venir que de la femme, l'homme peut faire échouer la conception d'un enfant si ses fonctions érectile et éjaculatoire sont défaillantes.

\section{L'impuissance des hommes}

Une impuissance par défaut permanent d'érection est nommée yò-kiidga, « verge morte ». Elle se distingue d'une autre par perte d'érection lors de la pénétration, appelée loèèga, " attachement ». Une impossibilité d'érection peut aussi être une forme mineure du pog-beodgo, littéralement «femme- piégée ». Enfin, existe une anéjaculation, raokoèènga, «l'homme sec».

Yò-kiidga et rao-koèènga sont des wend-banse, des maladies conformes à l'ordre du monde tel que l'être suprême (Wende) l'a créé, et donc pensées comme naturelles. Mais l'inscription dans cette catégorie, qui identifie d'office la cause efficiente, n'empêche pas de s'interroger pour savoir comment et pourquoi (causes instrumentale et ultime) la maladie survient (Zempleni, 1985). Lorsque yò-kïidga est primaire (aucune érection n'ayant jamais été possible), une décision de Wende, sans mobilisation d'une autre cause, est parfois évoquée. Mais, souvent, l'événement est imputé à l'écoulement de lait maternel ou d'une décoction utilisée par les femmes lors du maternage (voir plus loin) sur le sexe de cet homme lorsqu'il était bébé. Une autre cause est le contact de la verge avec le clitoris d'une femme incomplètement ou non excisée. Enfin, le fait qu'un enfant se soit assis (par inattention des femmes) sur l'une des pierres du foyer de la cuisine est aussi susceptible d'expliquer la survenue de cette impuissance.

Les autres sont perçues comme le résultat d'un acte humain volontaire. Loèèga est provoquée en déposant une préparation en un lieu que l'homme est susceptible d'enjamber, l'un des objectifs étant de fournir à une femme un argument de rupture d'alliance. Pour pog-beodgo, l'acte repose sur la crainte d'un possible adultère. Un homme qui pense que sa femme a un amant, ou qui veut se prémunir contre cette éventualité, peut préparer un " piège » qui rendra malade tout homme ayant un rapport sexuel avec elle.

\section{Page 48}


Interprétation religieuse et symbolique d'une stérilité

Dans la pensée moaaga existe une notion de prédétermination divine (pulemdé) définie par Wende pour de nombreux événements, en particulier le nombre d'enfants (rogem). Ainsi, est-il concevable qu'une femme ne puisse plus avoir d'enfants par épuisement de ce potentiel, nommé aussi yodre (nodosité, bosse, grumeau) et lorsqu'une mère allaitante est enceinte avant le sevrage, il est dit qu'elle a "volé le yodre » d'une autre femme ${ }^{5}$.

La Terre (Tenga) soeur et épouse de Wende, mère des génies et des hommes (Schweeger-Hefel, 1986), a aussi le pouvoir, par le biais des « autels de la Terre » (tenkuga), d'agir sur la capacité d'une femme à procréer. Les Moose disent, parlant d'un individu, qu'il est « l'enfant de tel ten-kugri », qui l'accompagne de la naissance à la mort, sans que cette parenté ait été forcément recherchée (Egrot, 2001). Mais, en cas de stérilité, la protection peut être demandée par des offrandes, imposant en cas de succès et pour que l'enfant survive, l'attribution d'un nom lié à l'autel sollicité.

Toujours en référence à la dimension symbolique de la procréation, une stérilité s'explique parfois par une difficulté de la femme à « séduire " un génie en brousse et des recommandations lui sont faites pour augmenter ses chances de ramener dans sa maison le génie qui servira de substrat à la conception d'un enfant (Bonnet, 1988, 1994 ; Lallemand, 1978).

\section{Perturbation du cycle menstruel, stérilité et fausse-couche}

Des anomalies du cycle menstruel peuvent aussi expliquer une impossibilité de procréer. Une interprétation de ces perturbations se réfère au ménage que chaque femme réalise quotidiennement dans sa case. Le balayage se fait du fond vers la porte, et la poussière doit aussitôt être ramassée. Toute négligence entraîne une perturbation du cycle menstruel. Ainsi, si une femme laisse la poussière à côté de la porte, elle risque d'avoir des règles longues et abondantes. Cette pensée métaphorique qui associe le corps et la maison ${ }^{6}$ ou les ordures et les menstrues, émerge

\footnotetext{
${ }^{5}$ II peut s'agir d'une co-épouse comme cela est décrit dans d'autres sociétés (Fainzang et Journet 1988), mais ce « vol » peut également affecter d'autres femmes, alliées à un autre buudu (segment de lignage) résidant dans le même village.

${ }^{6}$ Sur ce lien entre corps et maison, voir Fainzang (1986) qui analyse ce fait chez les Bisa du Burkina Faso à propos de la maladie des oiseaux (beno). Pour la maladie de l'oiseau chez les Moose et les rapports entre le corps et la maison, voir plus loin ainsi que Bonnet $(1988,1999)$ et Egrot (2001).
}

\section{Page 49}


aussi dans le registre thérapeutique. Ainsi, le fait de balayer de la porte vers le fond pendant quatre jours ${ }^{7}$ est un soin par inversion prescrit aux femmes ayant présenté des fausses couches. Néanmoins, ce traitement n'agit pas directement sur les désordres du corps mais permet de modifier la périodicité du cycle menstruel pour le remettre en phase avec le cycle lunaire. Cette discordance entre cycles menstruel et lunaire fournit une autre cause des troubles évoqués ici. D'autres traitements permettent d'ailleurs de rétablir cette nécessaire coïncidence entre les deux cycles, c'est-à-dire ramener la période menstruelle de la « mauvaise lune » vers la « bonne lune » (Egrot, 2001).

Les enfants « nés pour mourir »

La mort d'enfants avant l'âge de deux ans est aussi considérée comme une infécondité des femmes. Lorsque ces décès sont répétés, ils sont interprétés dans le registre symbolique, soit comme la conséquence d'une mort accidentelle d'animal provoquée antérieurement par l'un des habitants de la cour, soit comme une volonté des génies de voir l'enfant revenir avec ses parents d'origine ${ }^{8}$. Les enfants qui naissent ensuite sont appelés rogd-n-kiidsi, " nés pour mourir », et doivent, pour survivre, porter un prénom ayant pour racine le nom de l'animal décédé ou évoquant la pratique mise en oeuvre pour interrompre l'enchaînement des décès' .

L'ancêtre qui transmet le sèègre peut aussi être incriminé par l'envoi d'un « sèègre mortifère » (Bonnet, 1994). Mais alors, l'infécondité n'affecte pas une seule femme mais plusieurs, les décès concernant alors des germains et pas seulement des frères utérins. Pour le sèègre mortifère, une faute dans la famille du mari est évoquée ; pour les rogd-nkiidsi, une faute de la mère intervient dans le modèle explicatif (Bonnet, 1988).

Les représentations de la procréation et de ses éventuelles perturbations présentent donc une asymétrie entre les deux sexes. L'implication des hommes s'inscrit dans le physiologique ; celle des femmes, en grande partie, dans le symbolique et le religieux. Ce constat se trouve renforcé par une asymétrie des registres étiologiques mobilisés pour chacun des deux sexes lors des troubles de la reproduction. Commence ainsi à se dessiner

\footnotetext{
${ }^{7}$ Le chiffre 4 est la marque numérique du féminin, le 3 , celle du masculin. À ce propos, voir la note de bas de page $n^{\circ} 17$.

${ }^{8}$ Ces enfants qui meurent et qui reviennent sont nommés yewaya, soit littéralement : « il va revenir » (Bonnet, 1988, 1994).

${ }^{9}$ Pour des détails sur ces pratiques, voir Bonnet $(1988,1994)$ et Egrot (2001).
} 
une logique selon laquelle les hommes s'enracinent principalement dans le social, le profane ou le corporel, alors que les femmes se retrouvent plus facilement ${ }^{10}$ et souvent, dans le champ du religieux, en lien avec les êtres sacrés, la brousse ou l'environnement naturel. Une femme qui fait des fausses couches, appelée pu-yit-sooba (grossesse/ sortir/propriétaire), est perçue comme dangereuse, son infécondité mettant doublement en danger la fertilité du groupe. La mère d'une femme qui vit cette situation explique qu'" après une fausse-couche, une femme ne doit pas porter un enfant qui n'a pas de dent car la maladie qu'on appelle kaam peut l'attraper et aboutir à son décès ". Mais cette perception d'une propension des femmes à rendre malade ou à tuer des enfants dans le lignage du mari dépasse le cadre de cette situation spécifique.

\section{Maladies des enfants}

L'étiologie des maladies (et donc des décès) qui affectent les enfants se caractérise par une implication prépondérante des femmes. La société exige d'elles une attention constante jusque dans les faits en apparence les plus anodins. Si chacun sait que tout acte peut se révéler dangereux pour sa propre santé, les femmes ont en plus le lourd fardeau d'être souvent

\footnotetext{
${ }^{10}$ Cet ancrage spatial des hommes et des femmes est présenté ici plus comme une tendance dominante que comme une insertion spatiale systématique. Cela ne signifie pas que les informations ethnographiques recueillies à propos de la procréation puissent permettent de douter. Jamais, par exemple, ne fut évoqué un élément masculin qui puisse intervenir directement sur la fécondité d'une femme (comme c'est le cas pour le lait, le liquide de lavement, le clitoris ou la pierre du foyer, éléments de l'univers féminin dont le simple contact peut rendre un homme impuissant). De la même manière, aucune interprétation d'une impuissance faisant intervenir un être du monde religieux ou de l'univers naturel, ne fut exprimée en ma présence. La possibilité d'une infécondité féminine provoquée intentionnellement par un homme au moyen d'un acte de sorcellerie instrumentale, même si elle est en principe envisageable, ne fut jamais une hypothèse étiologique mobilisée en pratique. II existe néanmoins deux registres étiologiques communs aux deux types de perturbations et donc des intersections des deux espaces. Une infécondité ou une impuissance peuvent être considérées comme la conséquence d'une volonté de Wende et donc perçues comme naturelles. Des interprétations psychologiques ou biomédicales d'une infécondité féminine ou d'une impuissance sont parfois mobilisées de manière exclusive, mais par emprunt à un autre système de pensée.
} 
suspectées d'avoir occasionné des maladies chez les enfants par leur imprudence, leur négligence ou leur inconscience d'un éventuel danger.

\section{Les maladies attrapant l'enfant pendant la grossesse}

Par exemple, lorsqu'un décès survient dans le réseau social de la famille alliée d'une femme enceinte, le mari doit participer au creusement collectif de la tombe. Si un rapport sexuel a lieu lorsqu'il en revient, l'enfant à naître souffrira d'une maladie appelée yaoog-tom (yaoogo : tombe ; tom : terre). Ce nom repose sur une métaphore entre la poussière sur le corps du mari et le principal symptôme de l'affection qui fait «blanchir " l'enfant et sur un lien causal entre le creusement de la tombe et la maladie (11). Comme pour d'autres maladies survenant du fait d'un rapport sexuel, c'est la femme qui est désignée comme ayant failli à son obligation de retenue (Egrot, 2001).

Pour la maladie de "l'oiseau » (liuula), déjà décrite et analysée chez les Moose (Bonnet, 1986, 1988, 1999 ; Egrot, 2001), une métaphore s'établit en référence aux mouvements convulsifs et au cri émis parfois par l'enfant avant la crise, explicitement reconnus par les femmes comme évoquant le battement des ailes et le cri de l'oiseau. Mais le rapport est aussi causal, le survol de l'oiseau, son cri ou la superposition de son ombre avec celle de la femme enceinte déclenchant la survenue ultérieure de la maladie. Or, ce sont les femmes qui sont sensées éviter que de tels faits surviennent.

D'autres maladies affectant les enfants suite à un événement lors de la grossesse existent et impliquent souvent la mère dans le modèle explicatif ${ }^{12}$.

\section{Les maladies attrapant l'enfant lors de l'accouchement}

Lors de l'accouchement, deux maladies peuvent « attraper » un nouveau- né. Mais le risque n'existe qu'en un temps déterminé et dans des conditions précises. Le moment critique se situe entre l'accouchement et

\footnotetext{
${ }^{11}$ Comme pour la maladie suivante, il s'agit d'une mise en contact symbolique et médiatisée entre le mort dans sa tombe et l'enfant dans le ventre de sa mère (pour plus de détails, voir plus loin et Egrot, 2001).

12 Malformations de l'enfant par mécontentement d'un génie, maladies ou malheurs affectant l'enfant du fait d'un contact de la mère avec un animal, ou encore du fait de la consommation de viande d'animaux sauvages par la femme enceinte (Bonnet, 1988 ; Egrot, 2001).
}

\section{Page 52}


la délivrance et les femmes insistent sur le fait que l'incident doit survenir " avant que le cordon ombilical ne soit coupé ${ }^{13}$, l'enfant toujours posé au sol et donc avant qu'il ne soit lavé. Si la mère tousse et si la prophylaxie immédiate (Egrot, 2001) n'est pas faite, l'enfant aura plus tard, et parfois jusqu'à l'âge adulte, une maladie nommée pog-rogdkosgo, " toux de l'accouchement », une toux chronique souvent nocturne. Par ailleurs, la matrone doit veiller à ce que le « sang ${ }^{14}$ de l'accouchement n'entre pas en contact avec les yeux de l'enfant qui présenterait alors une affection oculaire, moenem ${ }^{15}$, qui se manifeste par des paupières gonflées, des yeux qui " se couvrent de sang " et, parfois, un écoulement purulent. La prophylaxie de ces deux maladies utilise le sang d'un animal tué au marché et les femmes insistent sur les exigences de qualité, c'est-à-dire du sang «frais, rouge, indemne d'impureté ». Au contraire, le "sang » de l'accouchement est perçu comme regdo, c'est-à-dire « impur, sale, souillé ».

Or, ces maladies ne s'expliquent pas par une simple contamination des yeux ou des voies respiratoires par un sang "sale ». Sinon, pourquoi la maladie ne surviendrait pas une fois le cordon ombilical coupé ? Pourquoi l'accoucheuse, en contact avec ce sang, n'est-elle pas malade ? Le cordon ombilical agit en fait comme un conducteur qui autorise la rencontre du sang de la mère et celui de l'accouchement au niveau des yeux ou des voies respiratoires de l'enfant (Egrot, 2001).

Mais l'accouchement n'est pas la seule situation à risque pour l'enfant, ni le sang le seul fluide corporel féminin potentiellement néfaste puisque le lait maternel peut être aussi générateur de troubles chez le nourrisson.

Les maladies attrapant l'enfant lors de l'allaitement et du maternage

Si un écoulement de lait sur le sexe d'un garçon entraîne une impuissance, il peut aussi, lorsqu'il pénètre l'oreille d'un bébé allongé sur les cuisses de sa mère, provoquer des maladies de l'oreille. La qualité du lait (biisem) est aussi l'objet d'une attention constante des mères qui distinguent biis-songo (lait-bon) et biis-yooko (lait-mauvais), comprenant différentes variétés de laits altérés (Alfieri et Taverne, 2000 ; Taverne, 2000). Les femmes en connaissent les symptômes, les tests pour le confirmer et

\footnotetext{
${ }^{13}$ Traditionnellement, le cordon ombilical est coupé après la délivrance.

${ }^{14}$ Ziim : le sang et les liquides corporels considérés comme des dérivés.

${ }^{15}$ Les discours soulignent l'analogie entre les yeux de l'enfant et ceux d'un albinos, moeega et un lien entre moeenem et moeega est parfois évoqué.
}

\section{Page 53}


les traitements pour les soigner. Mais l'enfant présente alors des troubles comme un refus de téter, des vomissements ou un ballonnement abdominal ${ }^{16}$ (Alfieri et Taverne, 2000 ; Egrot, 2001 ; Taverne, 2000).

La période d'allaitement est aussi l'objet d'un interdit sexuel, commun à de nombreuses ethnies en Afrique. Divers auteurs ont montré que la crainte du contact entre deux liquides corporels se construit sur les représentations qu'en ont les populations, sur les logiques du même et du différent et sur la notion de souillure (Cros, 1990 ; Douglas, 1992 ; Héritier, 1996). Le sperme, déposé dans le ventre de la mère, dénature le lait et les femmes évoquent "les saletés qui proviennent du ventre et que l'enfant tète ". Dans ce cas, "l'enfant fait la diarrhée ", son corps " blanchit » et il maigrit. Nombre de femmes disent être en mesure, en voyant les selles caractéristiques du bébé, de savoir si la mère a, ou n'a pas, respecté l'interdit sexuel (Egrot, 2001).

Les rapports sexuels, après une certaine durée d'allaitement, peuvent être fécondants. Les femmes disent qu'une telle grossesse met en danger la vie de l'enfant qui allaite, mais aussi que le foetus en gestation est un enfant volé, destiné à une autre femme. Comme pour les fausses couches, les femmes mettent en danger la survie de leur propre enfant, mais aussi la fécondité du lignage du mari puisqu'elles empêchent d'autres épouses de ce lignage d'avoir une grossesse.

La société confie aussi aux femmes les soins de maternage, faits deux fois par jour de la naissance jusqu'au sevrage. La chaîne opératoire commence par un lavement rectal (leedo) avec une décoction (yaamde) qui sert ensuite pour une ingurgitation forcée (yunugri) suivie d'une toilette (pèkré). Pendant les premières semaines, un soin (piisgu) est destiné à protéger le ventre de l'enfant par une obstruction transitoire de l'orifice tant que la « bouche du nombril » n'est pas totalement fermée (Egrot, 2004).

Certaines maladies, perçues comme issues d'une erreur lors du maternage, peuvent atteindre le bébé mais aussi des enfants plus grands, voire les adultes. Kibga survient lorsque les lavements sont mal faits, provoquant douleurs abdominales et amaigrissement. L'impuissance d'un homme est parfois imputée à l'écoulement du yaamde sur son sexe lorsqu'il était enfant. Enfin, deux maladies peuvent atteindre l'ombilic si de l'eau, de l'air ou des « saletés » pénètrent dans l'orifice ombilical (Egrot, 2001).

Les femmes sont donc presque systématiquement incriminées en cas de maladie chez les enfants. Mais cette mise en cause des femmes ne s'ar-

\footnotetext{
${ }^{16}$ Des maladies des seins peuvent également rendre l'enfant malade.
} 
rête pas aux frontières de cet espace puisqu'elles jouent également un rôle de premier plan dans la sorcellerie et dans la survenue de maladies affectant des individus adultes.

\section{Les actions humaines volontaires}

Une des causes évoquée, en cas de maladie, est l'action humaine volontaire. Deux types de sorcellerie existent. L'une est instrumentale et utilise un support matériel. L'autre est spirituelle, en ce sens qu'elle agit par capture d'une des composantes de l'esprit, le siiga. La désignation des auteurs d'un acte de sorcellerie établit cette distinction par l'usage des termes soeya et tiim-soaba (« possesseur de tiim ») ${ }^{17}$.

Les femmes sont suspectées en priorité de pratiquer la sorcellerie spirituelle. Cela exige d'avoir la capacité de voir le siiga, un don réservé aux personnes ayant des relations avec les mondes sacrés. En cas d'ingestion, la mort survient par « destruction métonymique ", la disparition du siiga "provoquant la destruction du sujet " (Bonnet, 1988). En cas de réclusion, la mort est différée et les conditions de séquestration sont réputées déterminer les symptômes (Bonnet, 1988). Cette sorcellerie est réputée avoir une sémiologie variée et se soigne plus difficilement.

La sorcellerie instrumentale est plus souvent attribuée aux hommes et utilise un principe actif, appelé tiim ou wak. La préparation ou l'objet efficient atteint sa victime par ingestion, contact, enjambement ou piétinement. Les Moose se méfient donc d'une simple noix de cola, d'une boisson ou d'un objet en travers du chemin. La sorcellerie instrumentale se traduit souvent par la survenue de maladies ayant une sémiologie déterminée (Egrot, 2001).

Mais d'autres actions humaines se rapprochent de cette sorcellerie. L'une est une thérapeutique qui permet de transmettre son mal à un autre pour pouvoir guérir. La seconde est un piège (begdo) qui protége les biens ou les personnes et a le pouvoir de rendre malade l'auteur d'un acte délictueux. Enfin, la malédiction (kaabgo) est utilisée en cas de litige entre deux personnes, celle qui s'estime lésée pouvant agir sur celle qu'elle accuse d'avoir commis un acte répréhensible à son égard. Les frontières entre sorcellerie instrumentale et autres actions humaines volontaires sont particulièrement floues et la légitimité d'un acte intentionnel à l'aide d'un tiim dépend étroitement des liens du locuteur avec l'agresseur ou l'agressé.

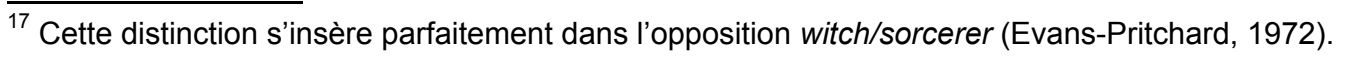


Cette répartition sexuée des deux types de sorcellerie confirme que les femmes sont plus facilement associées au monde de l'invisible et du sacré (ici, celui du siiga et donc des ancêtres) et sont, encore une fois, perçues comme éventuellement destructrices de l'un des symboles de la reproduction du lignage allié. Mais les femmes ont également le pouvoir de rendre les adultes malades et, pour l'illustrer, un retour sur la catégorie des "maladies de femmes " qui a permis d'introduire cet article, est nécessaire.

\section{Les " maladies de femmes »}

Une dizaine de maladies sont classées dans une catégorie nommée pug-banse, "maladies de femmes ». Certaines ont une sémiologie génitale ou urinaire, mais la sémiologie peut se localiser en d'autres endroits du corps ${ }^{18}$. Ainsi Sabga, une entité complexe, inclut de nombreux tableaux sémiologiques (diarrhée, vertiges, lombalgies, dysurie, etc.). Pug-konsgo, "femme-toux », est contracté lorsqu'une toux survient pendant le coït et se manifeste dans un délai variant de quelques mois à trois ans par une toux chronique avec des expectorations blanchâtres comparées au sperme, par la suite d'hémoptysies et la mort survient après deux à trois ans d'évolution. Pug-ruubo, «femme-morsure », survient suite à une morsure pendant le coït et se traduit par une "folie " après une phase silencieuse de un à quatre ans. Ku-pogdo, "femme de funérailles ", affecte tout homme qui aurait eu un rapport sexuel avec une veuve avant que celle-ci ne réalise le rituel de levée de deuil. Elle se manifeste par des oedèmes des pieds, des mains et des yeux et pourrait évoluer vers une lèpre (waoodo).

L'un des éléments communs de ces maladies est le fait qu'elles peuvent toutes se transmettre lors d'un rapport sexuel, même si certaines ont aussi d'autres modes de transmission. Ce critère d'inclusion, bien que nécessaire, n'est pas suffisant, d'autres maladies ayant une transmission sexuelle sans être des pug-banse. Le nom de la catégorie sous-entend que ces maladies viennent des femmes, notion qui se confirme dans les logiques d'imputation orientées principalement vers celles-ci. Mais cette asymétrie n'est pas non plus spécifique de cette catégorie. De plus, l'implication des femmes est très variable selon les pug-banse et, pour certaines, les hommes peuvent aussi être reconnus comme vecteurs éventuels, puisque seules deux pug-banse ne sont transmises que par les

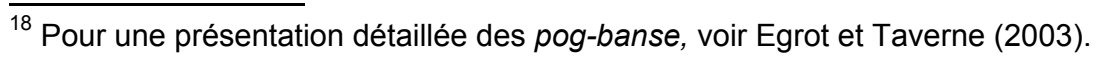


femmes. L'autre point commun entre plusieurs de ces maladies est le discours sur la gravité et la rapidité d'évolution, présentées comme plus importantes pour les hommes que pour les femmes. Mais là aussi, ce critère n'est pas applicable à l'ensemble de la catégorie $^{19}$.

En revanche, le nom de cette catégorie témoigne du fait que la parole valorisée en société moaga est celle détenue et produite par les hommes. L'appellation « maladies de femmes " et, plus globalement, l'asymétrie des registres étiologiques ne peut, en effet, se comprendre qu'en référence aux logiques sociales dans lesquelles elles s'insèrent.

\section{La maladie, lieu d'expression de la différenciation des sexes}

\section{Une interférence sur un large éventail de la nosographie}

Une corrélation entre sexe et maladie existe donc dans plusieurs espaces du champ de la santé et sur un large éventail de la nosographie populaire, que ce soit en termes de sémiologie ou de fréquence des événements. Cette interférence était peut-être prévisible pour les maladies liées à la reproduction. Les différences physiques entre hommes et femmes, leurs rôles biologiques respectifs dans la procréation, l'allaitement ou la sexualité, fournissent en effet des éléments d'observation concrets, induisant des processus cognitifs qui permettent l'appréhension de la réalité et l'élaboration de la pensée (Héritier, 1996). Néanmoins, il serait logique dans une telle dialectique que, s'éloignant des questions de reproduction, l'interférence se dilue jusqu'à s'estomper totalement. Or, il n'en est rien.

Certes, la proximité de la mère avec ses enfants pourrait justifier parfois son implication accidentelle dans la survenue d'une maladie chez l'un d'entre eux. Mais, si la quasi-totalité des maladies affectant la dernière génération d'un lignage implique surtout les femmes, le doute n'est plus permis sur le caractère social et culturel de cette logique causale. De plus, certaines maladies survenant chez des adultes sont aussi attribuées à la pratique des soins lorsqu'ils étaient enfants, extension de cette logique impliquant les femmes au-delà de la catégorie des maladies d'enfants. Les "maladies de femmes", qui ont une sémiologie variée, témoignent de cette implication préférentielle des femmes dans l'explication de maladies

\footnotetext{
${ }^{19}$ Force est de conclure (Egrot et Taverne, 2003) qu'il s'agit d'une classification polythétique (Vinéis, 1992).
} 
affectant des adultes. Le rôle différent de chaque sexe dans l'explication causale apparaît aussi pour la sorcellerie. Enfin gendba, la variole, nommée naaba, "chef », fut conçue jusqu'à son éradication comme "la plus puissante des maladies " du fait de sa sémiologie spectaculaire et de sa gravité. Or, certaines activités féminines étaient réputées attirer l'épidémie sur le village (Egrot, 2001).

Au-delà de la variété sémiologique, la fréquence des faits incriminés permet aussi d'affirmer que l'interférence entre maladie et sexe social est une réalité très présente au quotidien. Les maladies d'enfants concernent une tranche d'âge importante puisque environ un tiers de la population a moins de dix ans. Avec un taux de mortalité infantojuvénile entre $186 \%$ et $250 \%$ dans les vingt dernières années, les décès d'enfants avant quatre ans sont loin d'être des événements exceptionnels. Enfin, de nombreuses maladies dont les représentations interfèrent avec le sexe social, ont des prévalences suffisamment élevées pour être une préoccupation constante. Le sida à lui seul affecte, selon les estimations, $6,5 \%$ à $10 \%$ de la population en 2003. Mais les diarrhées, les infections respiratoires, le paludisme, pour ne citer que quelques exemples ayant une prévalence élevée, sont aussi responsables à eux seuls d'un nombre important d'épisodes de maladies et de décès qui s'insèrent dans des entités nosographiques populaires pour lesquelles une interférence avec le sexe social a été constatée. Ainsi, que ce soit sur la base de la variété des tableaux sémiologiques ou sur celle de la fréquence des maladies incriminées, l'interférence entre maladie et sexe social apparaît comme une réalité bien établie et largement partagée ${ }^{20}$.

\section{Asymétrie de la sémiologie, des registres étiologiques et de l'imputabilité}

La mobilisation du sexe social dans les représentations des maladies s'effectue de différentes manières. L'interprétation de la sémiologie et le diagnostic ne dépendent souvent pas du sexe de l'individu, sauf pour des maladies spécifiquement féminines ou masculines du fait des différences biologiques entre hommes et femmes ou de quelques cas qu'il serait trop long de développer ici. En revanche, une différenciation sexuelle très nette

\footnotetext{
$\overline{20}$ II existe également des asymétries dans le registre thérapeutique, en particulier par l'usage du 3 ou du 4, chiffres symboliques du masculin et du féminin (tarifs des médicaments, nombre d'objets composant le traitement, nombre d'actes à réaliser, etc.). Cette différenciation sexuée des traitements à déjà été analysée dans d'autres sociétés (Fainzang, 1985) et également chez les Moose (Egrot, 2001). Par nécessité de synthèse, cet aspect de la question n'a pu être traité dans le présent article.
} 
existe dans les discours sur la sémiologie pour les " maladies de femmes " puisque certaines sont présentées comme ayant une expression clinique et une évolution différentes selon qu'il s'agit d'une femme ou d'un homme. Mais les différences sont surtout évidentes au niveau de la causalité : d'une part, les registres étiologiques peuvent être différents selon que la maladie affecte une femme ou un homme, d'autre part, certaines maladies sont plus spécifiquement imputées à l'un ou l'autre sexe. II y a donc une asymétrie des registres étiologiques selon le sexe de l'individu malade ou une asymétrie sexuelle de l'imputabilité.

L'analyse des troubles de la reproduction a montré une nette différence des registres d'interprétation mobilisés. Pour les femmes, les causes efficientes évoquées sont pour l'essentiel des acteurs d'ordre naturel (lune, animaux) ou religieux (Wendé, tenkuga, kinkirse, ancêtres). En regard, les différentes formes d'impuissance s'expliquent, outre les causes imputables à Wendé, par des interventions humaines. Au niveau de la cause ultime, l'asymétrie des registres étiologiques se confirme. Certaines impuissances surviennent parce qu'une femme transgresse des règles de l'alliance (adultère, séparation) ou qu'elle a mal réalisé les soins ou la surveillance des hommes lorsqu'ils étaient enfants. En revanche, les perturbations de la fécondité sont rarement interprétées comme la conséquence d'un désordre du social mais bien plus souvent comme une perturbation de l'équilibre entre monde humain et environnement naturel ou religieux.

L'asymétrie sexuelle de l'imputabilité apparaît dans la recherche de la cause efficiente orientée surtout vers les femmes pour la sorcellerie spirituelle et les hommes pour la sorcellerie instrumentale. L'analyse a montré que la première est conçue comme surnaturelle, nécessitant le don de voir le siiga, et témoigne d'une volonté délibérée de faire du mal. Elle se traduit de manières variées et peut donc se dissimuler derrière toutes entités nosographiques. Au contraire, la seconde, plus humaine, plus sociale, peut parfois être perçue comme la solution d'un conflit. La frontière avec d'autres actions humaines volontaires totalement légitimes apparaît alors particulièrement floue. Enfin, elle se manifeste le plus souvent par des tableaux sémiologiques déterminés correspondants à des entités nosographiques définies.

Pour les « maladies de femmes », l'asymétrie sexuelle de l'imputabilité apparaît dans les explications fournies à propos de la transmission. Deux d'entre elles ne sont transmises que par les femmes. Mais, dans le modèle explicatif de la plupart d'entre elles, les femmes continuent d'être présentées comme à l'origine du mal. Le rapport sexuel apparaît alors comme un acte particulièrement dangereux pour les hommes et, lorsque la recherche d'une cause ultime est effectuée, c'est essentiellement l'adultère ou une transgression d'interdit des femmes qui sont évoqués. 
Enfin, pour la quasi-totalité des maladies et des décès affectant les enfants, les femmes sont désignées comme causes instrumentales ou efficientes, du fait d'une imprudence, d'une négligence ou d'une volonté délibérée de nuire. Si elles sont indispensables et seules responsables de la production d'une descendance pour le lignage du mari, elles sont aussi les premières à pouvoir occasionner son altération, voire sa destruction.

\section{Représentations des sexes et logiques sociales mobilisées}

L'interférence entre sexe et maladie étant établie, son étendue circonscrite, les niveaux de causalité dans lesquels elle se déroule précisés, restent à déterminer les représentations du sexe et les logiques sociales mobilisées en de pareilles occasions.

Comprendre l'identité masculine des Moose impose une référence au patrilignage et au territoire correspondant, en raison des principes de patrilinéarité et de virilocalité qui régissent la gestion du patrimoine, l'alliance matrimoniale et la résidence. Ces principes induisent une conception segmentaire, délimitant des unités discrètes, généalogiques, territoriales et sociales (Izard, 1985), ainsi qu'une fixité territoriale. Les hommes sont chargés d'assurer l'unité, l'harmonie, la protection du lignage, mais aussi la disjonction entre univers sacrés et monde profane21. Nombre de cérémonies coutumières visent, en effet, à maintenir cette séparation. Les agnats réalisent les actes ayant pour but de disjoindre un nourrisson de son " frère " génie, alors que la mère met tout en oeuvre pour ne pas contrarier les kinkirse (Bonnet, 1988 ; Egrot 2004 ; Lallemand, 1977, 1978). Les hommes se chargent, lors des funérailles, des rituels visant à garantir le départ et le voyage de l'esprit du mort afin qu'il n'importune pas les vivants (Raynal, 1995). Ce sont eux aussi qui remercient les ancêtres pour les récoltes sans en oublier un seul, omission qui aurait pour effet la survenue d'un malheur par mécontentement d'un aïeul.

Ce caractère disjonctif des hommes s'exprime aussi dans les incestes symboliques frères/soeurs de la mythologie et des contes, qui révèlent un rêve d'autonomie totale du lignage (Egrot, 2001). Néanmoins, un groupe agnatique, fixé dans son lieu de résidence, verra ses filles partir et des épouses arriver.

À l'inverse, les femmes n'ont pas de territoire fixe. Lors du mariage, elles quittent la terre paternelle pour une autre, où elles seront considérées comme étrangères. L'un des termes désignant une femme qui arrive est d'ailleurs saana, l'étrangère. Elle ne fera partie du lignage de son mari

${ }^{21}$ Cette conclusion rejoint celle de Hicks pour les Baruya (Hicks, 1985).

\section{Page 60}


qu'après des années de vie conjugale et à condition qu'elle lui ait donné des enfants qui, quoi qu'il arrive, appartiennent au lignage du père dans le droit coutumier. Mais toute femme, épouse puis mère en un lieu, reste soeur ailleurs. L'identité féminine valorisée dans la mythologie et les contes est celle de la soeur, alors que les épouses, si elles apparaissent, sont présentées comme égoïstes, peu soucieuses de faire le bien, avec une attitude d'opposition. Les étapes de l'alliance soulignent aussi la cohésion et la force du lignage du mari, et les relations à plaisanterie apparaissent dès qu'une femme doit quitter ou pénétrer un lignage, pour la retenir dans le premier cas, lui interdire l'accès à sa future cour dans le second (Egrot, 2001).

La double identité territoriale des femmes a pour corollaire une plus grande mobilité (Maïzi, 1995 ; Vinel, 1998). Si le discours fait état d'une obligation de rester dans le village du mari et que tout départ est souvent décidé après autorisation de ce dernier, les femmes séjournent en réalité bien plus que les hommes dans d'autres lieux que leur village d'alliance. Aucun homme ne peut refuser à une épouse d'aller dans son village paternel ou dans un lieu où se trouve l'une de ses tantes ou de ses soeurs qui sont dispersées dans des villages différents toujours en raison de la règle de patri-virilocalité, car elle a le droit, et même le devoir, de rendre visite à ses parents ${ }^{22}$.

L'alliance matrimoniale et la mobilité des femmes sont des réalités sociales grâce auxquelles se créent les liens entre les diverses unités lignagères. Une unité territoriale se trouve en relation avec une multitude d'autres par l'intermédiaire des tantes paternelles, des soeurs et des filles (logique agnatique), mais également par les épouses et les tantes maternelles (logique de l'alliance). Les règles qui régissent les cérémonies coutumières rappellent d'ailleurs sans cesse les liens tissés grâce aux femmes, que ce soit lors des funérailles, des étapes de l'alliance ou encore des naissances.

Cette fonction conjonctive des femmes ${ }^{23}$ dépasse le domaine territorial et social. Par la procréation, elles apparaissent comme le point de convergence des espaces profane et religieux définis par la culture puisque les génies et les ancêtres se rejoignent dans le ventre des femmes pour créer à un nouvel être humain avec l'aide des deux divinités, des autels

\footnotetext{
${ }^{22}$ Plusieurs cérémonies coutumières imposent que les filles ou les soeurs soient présentes : funérailles, alliance matrimoniale, naissance, maladie, ainsi que bien d'autres situations (Egrot, 2001 ; Raynal, 1995 ; Vinel, 1998).

${ }^{23}$ Sur l'opposition entre disjonction masculine et conjonction féminine, voir Hicks (1985).
}

\section{Page 61}


chthoniens, parfois des animaux et de l'environnement naturel. L'opposition femmenature/homme-culture (Mathieu, 1973) trouve donc ici une nouvelle expression. Mais les femmes apparaissent aussi comme le ciment permettant l'assemblage des différentes unités territoriales et sociales, et aussi des différents mondes qui composent l'univers moaaga. Les femmes ont, en reprenant ici l'analyse de Hicks, le " pouvoir de médiation » et le « pouvoir d'intégration ». Elles assurent la liaison entre le lignage par l'alliance et la rencontre entre univers sacrés et monde profane par la procréation.

Si les femmes sont perçues proches de la nature et que les hommes s'arrogent le contrôle de la culture, elles sont aussi perçues par les seconds comme des êtres surnaturels, capables de maîtriser, par la procréation, les différentes forces de la création. Dans la logique masculine, cette puissance sur-naturelle des femmes doit, comme les divinités, les autels de la terre, les génies ou les ancêtres, les éléments naturels (le feu, la foudre, le vent, etc.), être domestiquée et maîtrisée par les hommes. Le paradoxe de la féminité est alors évident. Une épouse arrive dans le village de son mari comme une étrangère dont il faut se méfier. La terre qui l'accueille n'est pas celle de ses ancêtres ; elle n'y possédera jamais rien. Mais elle est, par ailleurs, seule capable de reproduire le groupe familial et cette fonction la positionne en marge des hommes, à l'interface entre monde humain et mondes sacrés, entre le village et la brousse. La multi-territorialité, la mobilité qui en découlent et cette position de point focal de la culture, sont les principales logiques sociales et culturelles qui fondent les représentations de la féminité.

\section{Mobilité des femmes dans la gamme des valeurs symboliques}

Si la fixité territoriale des hommes s'oppose à la mobilité des femmes, le caractère disjonctif des premiers au caractère conjonctif des secondes, l'enracinement social des uns aux liens étroits des autres avec la nature et les puissances sacrées, le sexe social est aussi un mode de déclinaison des représentations du corps et de la physiologie.

S'il est classique d'associer l'opposition féminin-masculin aux autres séries de valeurs oppositionnelles comme le chaud et le froid, l'humide et le sec ou la droite et la gauche, ces associations ne sont pas systématiques dans la société moaaga. Elles existent certes pour quelques cas, puisque le sucré, la fertilité, l'humide, ou encore l'Est sont plus spécifiquement liés au féminin.

Ce qui oppose féminin et masculin, c'est surtout l'extrême mobilité des femmes dans la gamme des valeurs symboliques, notamment entre la 
puberté et la ménopause. Selon la phase de leur cycle menstruel ou leur état obstétrical, elles seront tantôt associées au froid, tantôt au chaud. Selon l'état d'avancement d'une grossesse, l'alimentation sera modifiée, autorisant les mets sucrés puis les interdisant, prescrivant l'usage exclusif de la potasse dans les sauces, du chaud et du cuit en fin de grossesse ou après une fausse couche. Pendant l'allaitement, d'importantes variations existent, le lait étant quelquefois perçu comme trop amer, trop sucré, trop fade, etc., imposant à la mère une constante adaptation de son alimentation. L'opposition droite/gauche ne subit que rarement une déclinaison masculin/féminin. Elle sert plutôt à opposer la vie et la mort, le monde humain et celui des génies. Ainsi, la mobilité des femmes dans la gamme des valeurs symboliques rejoint celle déjà signalée dans d'autres registres. Ces changements répétés de référents symboliques augmentent le risque de devenir malade au contact des femmes, puisque la rencontre du même ou du différent génère un déséquilibre, source de désordre. Cette mobilité des femmes participe donc pleinement à la construction culturelle de la notion de dangerosité du féminin.

Cette notion est bien marquée pour la sexualité, lieu de rencontre nécessaire entre les sexes, qui apparaît plus dangereuse pour les hommes que pour les femmes. La rencontre des corps doit donc être la plus brève possible et suivre des règles présentées comme très strictes pour minimiser les risques d'altération de soi (Egrot, 2001). Pour le patrilignage, référentiel spatial et social de la pensée et de la parole, le coït est source d'altération des individus masculins par la survenue de pug-banse mais aussi par d'autres maladies provoquées par les contacts avec les menstrues ou le sperme d'un autre homme en cas de multiplicité des partenaires. Mais la nuisance du féminin s'exerce aussi en dehors de la sexualité, puisqu'il a la puissance de détruire l'efficacité des amulettes et des divers objets de protection du mari.

\section{De la souillure à la maladie}

Plusieurs modèles étiologiques mobilisent la notion de saleté, d'impureté (regdo) pour expliquer une maladie. Mais les éléments considérés comme souillures ne le sont pas toujours en permanence et pour tout le monde. Le passage d'une substance en un lieu non conforme à sa fonction et à sa destination habituelle, ou la mise en contact de deux substances identiques ou pensées comme telles, fait naître la notion de saleté à partir d'éléments qui ne l'étaient pas antérieurement. Ainsi, le sperme, le lait et le sang sont des liquides corporels qui, contrairement aux urines, aux excréments ou aux menstrues ne deviennent « sales » que dans des

\section{Page 63}


circonstances précises. De même, la poussière de tombe n'est pas dangereuse pour toute personne et seuls les enfants en gestation y sont sensibles.

Par exemple, le caractère ambigu du sperme apparaît dans une forme de pugpoaaga se traduisant par une masse sortant par la vulve, imputée à une accumulation abdominale de sperme, entré "par effraction " dans le ventre de la femme lors des rapports sexuels. L'analyse des explications montre que les termes utilisés pour désigner le sperme, tantôt valorisants, tantôt dépréciatifs, varient selon son emplacement dans le corps de la femme (Egrot, 2001). Dans le même ordre d'idée, toute émission de sperme en dehors du vagin est conçue comme souillure. Pour le pug-konsgo, la toux induit un retour de sperme dans la verge ou une pénétration trop importante dans le corps de la femme, responsable ensuite d'expectorations comparées explicitement à du sperme. Pendant l'allaitement, le sperme altère le lait provoquant des diarrhées dans lesquelles les glaires blanchâtres sont assimilées au sperme contenu dans le ventre des femmes allaitantes. Le sperme est aussi incriminé dans la survenue de plusieurs pug-banse, notamment par la rencontre du sperme d'un autre.

Ainsi, la " souillure » apparaît si un élément porteur d'une valeur spécifique dans l'univers social ne respecte pas un ordre communément admis (Douglas, 1992), à savoir ici, dès que le sperme "n'est pas à sa place " d'un point de vue anatomique ou physiologique. Si le sperme quitte les voies génitales, lieu anatomique conforme à sa fonction, dès qu'il ne participe plus à la conception d'un enfant, il devient souillure et entraîne une maladie. De la même manière, la rencontre de spermes prouve que l'un des deux n'est pas à sa place, en raison des règles sociales qui régissent la sexualité.

Les femmes jouent donc un rôle essentiel dans l'émergence de la souillure. Leur corps est souvent le lieu de transformation d'un élément en saleté, le lieu de rencontre entre deux substances qui ne devraient pas se rencontrer. II en va ainsi du mélange des spermes, de la rencontre du lait et du sperme, du sang de l'accouchement avec les voies aériennes ou les yeux du bébé ou des "impuretés cadavériques " avec l'enfant en gestation. Mais les femmes sont aussi impliquées dans l'éclosion de la souillure par certains de leurs actes. Ainsi, la mauvaise réalisation des soins aux enfants, les erreurs lors de l'allaitement, la toux pendant l'accouchement ou le coït, la rupture d'interdit sexuel pendant le deuil ou l'allaitement, la négligence du balayage, l'adultère ou la multiplicité des partenaires, sont des occasions d'émergence d'une souillure susceptible de provoquer la survenue d'une maladie.

\section{Page 64}




\section{La conduction des maladies par les femmes}

La faculté des femmes à provoquer des maladies oblige aussi à revenir sur les notions de contagion, transmission et contamination, bien imparfaites pour traduire les conceptions émiques mobilisées dans certains modèles étiologiques. Ces insuffisances conceptuelles sont particulièrement flagrantes pour des maladies comme liuula, yaoogtom, moeenem ou pog-rogd-konsgo pour lesquelles le contact avec un agent causal ne suffit pas à expliquer la maladie. La menace qu'il représente n'est réelle que pour certains individus déterminés et dans des circonstances particulières.

La contiguïté a été définie comme "une identité proposée par la culture de deux choses perçues comme en relation proxémique». Agent causal et individu malade sont des "catégories pensées comme trop identiques (ou trop symétriques) pour être accolées sans risque de "courtcircuit" " (Jacob, 1988). La notion apparaît pertinente pour les exemples cités puisque les modèles explicatifs mettent en relation le mort dans sa tombe et l'enfant dans le ventre de sa mère, ou alors deux sangs contigus à travers le cordon ombilical. Mais une question persiste à laquelle ne répondent ni les théories de la transmission, ni le concept de contiguïté. Les vecteurs, tels la poussière de tombe, l'oiseau, son cri, le corps de la mère ou le cordon ombilical, occupent une place centrale dans la survenue des maladies évoquées. Ainsi, l'agent responsable ne peut être transmis que par des objets, des substances ou des êtres vivants bien déterminés, unis par des liens dont la nature reste à préciser. L'idée d'une conduction peut alors germer et se définir comme l'action de transmettre de proche en proche un agent causal matériel ou symbolique, entre deux objets ayant des polarités trop proches ou trop opposées dans le système des valeurs symboliques (rapport de contiguïté). Cette conduction induit la survenue d'une maladie dès lors que le contact est établi. Elle s'effectue au travers d'objets ou d'êtres disposant de propriétés et de positions bien déterminées dans l'univers culturel et social, ce qui leur confère alors le rôle de conducteurs de la maladie (Egrot, 2001).

Ce cadre conceptuel permet d'affirmer que les femmes sont conductrices pour nombre de maladies. Cette conductibilité féminine s'explique en partie par leur position, tant sociale que culturelle, à l'interface des divers mondes définis par la culture moaaga et par leur mobilité, notamment dans la gamme des valeurs symboliques

\section{Page 65}




\section{La maladie, espace de résonance et de légitimation de la différenciation des sexes}

L'implication des femmes dans l'émergence de la souillure et la conduction des maladies, la menace qu'elles représentent dans la pensée des membres du lignage allié, sont autant d'éléments qui renforcent la différenciation des sexes. Si un enfant est malade ou décède, lorsqu'un devin évoque une capture du siiga, lorsque des maladies affectent les hommes, les regards se tournent immédiatement vers les femmes pour identifier les causes de l'événement. Certes, elles ne sont pas nécessairement accusées d'avoir délibérément provoqué le mal. Hormis la sorcellerie, c'est bien plus souvent une négligence, une imprudence ou une faute qui permettent d'expliquer la survenue de la maladie. Mais les femmes sont néanmoins tenues à l'écart car elles peuvent nuire aux hommes et à leur descendance. Presque quotidiennement dans un village, la survenue d'une maladie et surtout l'explication fournie en apporte la confirmation. Cette nuisance potentielle apparaît comme un justificatif puissant d'une séparation tant spatiale que sociale entre les hommes et leurs alliées. Cet usage social de la maladie n'est certes pas le seul argument qui impose ce partage, mais il en constitue sans conteste l'un des principaux fondements.

Ainsi, les femmes vivent dans un espace bien séparé de celui des hommes. Si une femme est conviée à venir le soir dans la chambre de son mari, c'est, du moins en société traditionnelle, pour avoir une relation sexuelle brève obéissant à des règles strictes qui permettent de limiter les risques induits par cette nécessaire rencontre des deux sexes. Dans tous les autres actes de la vie quotidienne, un respect le plus strict possible de la frontière entre féminin et masculin est observé : hommes et femmes dorment dans des espaces différents, une femme ne peut partager un plat de nourriture avec un homme, hormis pour les cultures collectives, chaque sexe exécute les travaux qui lui incombent en des lieux le plus souvent différents.

Malgré cette séparation, la puissance des femmes inquiète toujours. Une femme qui a ses règles ne doit plus approcher la case de son mari ni aucun lieu sacré, car elle a alors le pouvoir de détruire la puissance des amulettes et des fétiches. Elle ne doit plus, en principe, préparer la nourriture, bien que cet interdit semble spécifique aux familles musulmanes dans la société moaga ${ }^{24}$. Le transport de feu d'un foyer à un autre, soit

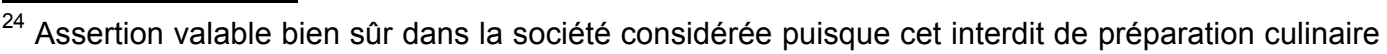
pendant la période de menstruation est largement répandu dans de nombreuses sociétés, sans qu'elles ne soient nécessairement islamisées (voir notamment Héritier, 1996)
}

Page 66 
d'une cour de femme vers celle de l'une de ses co-épouses est sévèrement réprimandé par les hommes car il est réputé détruire le pouvoir des amulettes de protection du mari. Enfin, "la bouche des femmes " est perçue comme dangereuse et bien des proverbes expriment cette crainte des hommes d'avoir à subir leurs "flèches » verbales ${ }^{25}$. Les risques de maladies occasionnées par les femmes viennent donc renforcer une représentation de la féminité déjà fortement présente par ailleurs.

La maladie est donc un lieux d'expression mais aussi un outil de consolidation, de la différenciation sociale et culturelle des sexes. Ainsi, quand la maladie se décline en référence au sexe, les accords obtenus résonnent dans le social pour confirmer leur nécessaire séparation. Si le sexe social structure les représentations de certaines maladies, en retour celles-ci légitiment cette différenciation.

\section{RÉFÉRENCES BIBLIOGRAPHIQUES}

Annandale E., Hunt K., 2000, Gender inequalities in health, Buckingham, Open University Press.

Alfieri C., Taverne B., 2000, Ethnophysiologie, règles et précautions chez les Madare et les Mossi / Ethnophysiologie des difficultés et complications de l'allaitement maternel / Perceptions de la transmission des maladies par l'allaitement maternel au Burkina Faso, In : Desclaux A., Taverne B., eds, Allaitement et VIH en Afrique de l'Ouest, Paris, Karthala : $135-188 ; 219-238$.

Badini A., 1978, Représentation de la vie et de la mort chez les Moose traditionnels de Haute-Volta, Thèse de Philosophie, Lille III.

Bonnet D., 1988, Corps biologique, corps social. Procréation et maladies de l'enfant en pays mossi, Burkina Faso, Paris, ORSTOM.

Bonnet D., 1994, L'éternel retour ou le destin singulier de l'enfant, L'Homme, 34, 3, 93110.

Bonnet D., 1999, Les différents registres interprétatifs de la " maladie de l'oiseau », In : Jaffre Y., Olivier de Sardan J.-P., eds, La construction sociale des maladies, Paris, PUF, 305-320.

Cros M., 1990, Anthropologie du sang en Afrique. Essai d'hématologie symbolique (Lobi, Burkina Faso, Côte-d'Ivoire), Paris, L'Harmattan.

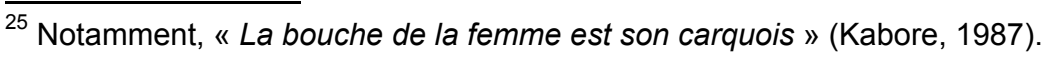

\section{Page 67}


Desclaux A., 1997, L'épidémie invisible. Anthropologie d'un système médical à l'épreuve du sida chez l'enfant à Bobo Dioulasso, Burkina Faso, Thèse d'anthropologie, Université d'Aix-Marseille.

Douglas M., 1992, De la souillure, Paris, La Découverte.

Duchesne V., 2003, Fluides, transmission et filiation. Les maladies de femmes dans une société matrilinéaire ivoirienne, In : Bonnet $\mathrm{D}$, Jaffre $\mathrm{Y}$., eds, Les maladies de passage, Paris, Karthala, 199-220.

Egrot M., 2001, La maladie et ses accords. Le sexe social, mode de déclinai - son et espaces de résonance de la maladie chez les Moose du Burkina Faso, Presses Universitaires du Septentrion.

Egrot M., 2004, La protection du ventre des bébés et la fermeture de la bouche du nombril (Burkina Faso), In : Bianquis I., Le Breton D., Méchin C., eds, Orifices du Corps, traversées anthropologiques, Paris, L'Harmattan (sous presse).

Egrot M, Taverne B., 2003, Représentations de la transmission sexuelle des maladies chez les Mossi au Burkina Faso ; rencontre des catégories nosologiques populaires et biomédicales dans le champ de la santé publique, In : Bonnet D, Jaffre Y., eds, Les maladies de passage, Paris, Karthala, 221-251.

Evans-Pritchard E, 1972, Sorcellerie, oracles et magie chez les Azandé, Paris, Gallimard.

Fainzang S., 1985, Les sexes et leurs nombres. Sens et fonction du 3 et du 4 dans une société burkinabé, L'Homme, 25 (4), 97-109.

Fainzang S., 1986, "L'intérieur des choses ». Maladie, divination et reproduction sociale chez les Bisa du Burkina, Paris, L'Harmattan.

Fainzang S., Journet O. 1988, La femme de mon mari. Étude ethnologique du mariage polygamique en Afrique et en France, Paris, L'Harmattan.

Fassin D., 1996, L'espace politique de la santé, Paris, PUF.

Héritier F., 1996, Masculin/Féminin. La pensée de la différence, Paris, Odile Jacob.

Hicks D., 1985, Conjonction féminine et disjonction masculine chez les Tetum, L'Homme, XXV (2), 23-36.

Izard M., 1985, Gens du pouvoir, gens de la terre. Les institutions politiques de l'ancien royaume du Yatenga, Cambridge, Cambridge University Press.

Jacob J.-P., 1988, Interprétation de la maladie chez les winye du Burkina

Faso. Critique d'une théorie de la contamination, Genève-Afrique, 59-88.

Kabore O., 1987, Paroles de femmes, Journal de la Société des Africanistes, 57(2), 117132.

Lallemand S., 1977, Une famille mossi, Paris, Ouagadougou, Recherches Voltaïques, 17.

Lallemand S., 1978, Le bébé-ancêtre Mossi, In : Système de signes, Paris, Hermann, 308-316. 
Maïzi P., 1995, Identités plurielles des femmes moose du Yatenga (Burkina Faso), Cahiers des Sciences Humaines, 31 (2), 485-499.

Mastro T.D., de Vincenzi I., 1996, Probabilities of sexual HIV-1 transmission, AIDS, 10, S75-S82.

Mathieu N., 1973, Homme-culture, femme-nature, L'Homme, 13, 3, 101-113.

Pollard T.M., Hyatt S.B., 1999, Sex, gender and health, Cambridge, Cambridge University Press, Biosocial Society Symposium Series, 11.

Rao Gupta G., Weiss E., 1993, Women's lives and sex: implications for AIDS Prevention, Culture, Medicine and Psychiatry, 17, 399-412.

Raynal M., 1995, Cérémonies funéraires dans le Zitenga (Burkina Faso), Thèse d'anthropologie, Université de Paris X.

Saillant F., 1991, Les soins en péril : entre la nécessité et l'exclusion, Recherches Féministes, 4, 1, 11-30.

Saillant F., 1992, La part des femmes dans les soins de santé, Revue Internationale d'Action Communautaire, 28, 66, 95-106.

Sargent C., Brettell C., 1995, Gender and health, Prentice Hall.

Schweeger-Hefel A., 1986, Kinkirsi-Boghoba-Saba. Das Weltbild der Nyonyosi in Burkina Faso, Wien, Verlag A. Schnendl.

Sweetman C., 2001, Gender, development and health, Oxfam.

Taverne B., 1996, Stratégie de communication et stigmatisation des femmes lévirat et sida au Burkina Faso, Sciences Sociales et Santé, 14 (2), 87-105.

Taverne B., 1999, Valeurs morales et messages de prévention : la " fidélité » contre le sida au Burkina Faso, In : Becker C., Dozon J.-P., Obbo C., Touré M., eds, Vivre et penser le sida en Afrique, Paris, Karthala, 509-525.

Taverne B., 2000, L'allaitement dans le cycle de vie de la femme en pays mossi / Les détenteurs du savoir et les alternatives à l'allaitement en milieu rural mossi, In Desclaux A., Taverne B., eds, Allaitement et VIH en Afrique de l'Ouest, Paris, Karthala, 83-110 ; 293-267.

Vinéis P., 1992, La causalité en médecine, Sciences Sociales et Santé, 10, 3, 5-32.

Vinel V., 1998, La famille au féminin. Société patrilinéaire et vie sociale féminine chez des Sikoomse (Moose, Burkina-Faso), Thèse d'anthropologie, EHESS.

Zempleni A., 1982, Anciens et nouveaux usages sociaux de la maladie en Afrique, Archives des Sciences Sociales des Religions, LIV (1), 5-19.

Zempleni A., 1985, La maladie et ses causes, L'Ethnographie, 2, 13-44

\section{Page 69}




\begin{abstract}
Sexual differentiation and causal interpretation of illness in subsaharian Africa (Burkina Faso)

The social sex is implied in the construction and representation of several illnesses among the Mossis of Burkina Faso, especially those regarding sexuality (diseases transmitted during sexual intercourse), individual reproduction (sterility, impotence) and group reproduction (children diseases and witchcraft). Interferences between these categories may exist and imply an asymmetry between etiological models and imputation logics. They also represent opportunities to enhance the social norms associated to each gender and the risks generated by transgression. The illness is both a form of expression and a space of legitimation of the social differentiation between genders
\end{abstract}

\title{
RESUMEN
}

\section{Diferenciación sexual e interpretaciones causales de la enfermedad en Burkina Faso}

El sexo social interviene en la construcción de la representación de varias enfermedades de los Mossi de Burkina Faso, en particular aquellas que conciernen a la sexualidad (enfermedades transmitidas durante la relación sexual), la reproducción individual (infertilidad, impotencia) y la reproducción del grupo (enfermedades de los niños, propiedades del patrilinaje y brujería). Cuando existen, estas interferencias se aducen principalmente por una asimetría en los modelos etiológicos y en las lógicas de imputación y son ocasiones en las cuales se hacen valer las normas sociales asociadas a cada sexo y a los riesgos engendrados por su transgresión. La enfermedad aparece entonces como uno de los lugares de expresión de esta diferenciación sexual pero también como un espacio de legitimación de la diferenciación social de los sexos. 